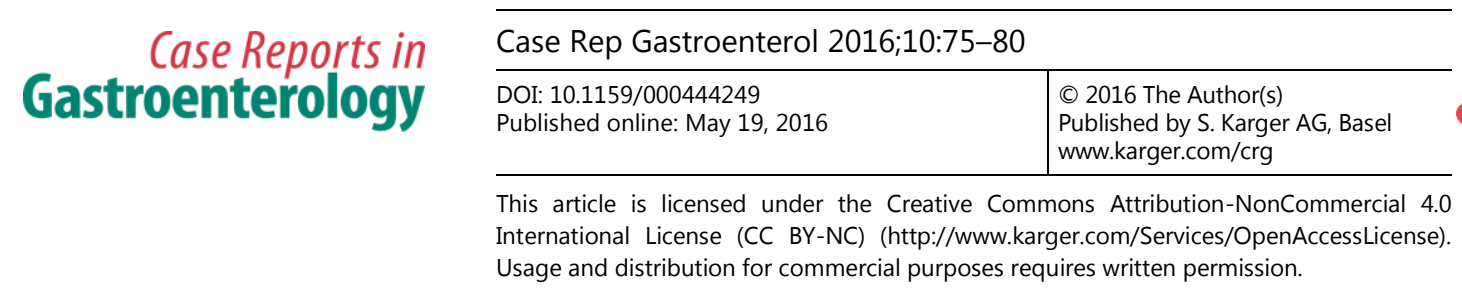

\title{
Hematogenous Gastric Metastasis of Pancreatic Cancer
}

\author{
Junpei Sasajima ${ }^{a, b} \quad K^{2}$ otaro Okamoto ${ }^{a} \quad$ Masato Taniguchi ${ }^{a}$ \\ ${ }^{a}$ Department of Gastroenterology, Ogasawara Clinic, Sapporo Hospital, Sapporo, Japan; \\ ${ }^{b}$ Division of Gastroenterology and Hematology/Oncology, Department of Medicine, \\ Asahikawa Medical University, Asahikawa, Japan
}

\section{Keywords}

Pancreatic neoplasm · Neoplasm metastasis · Stomach · Endoscopy · Digestive system

\begin{abstract}
While the gastric involvement of pancreatic cancer is occasionally observed as the result of direct invasion, hematogenous gastric metastasis is rare. A 72-year-old Japanese male presented with general fatigue, pollakiuria, and thirst. Computed tomography revealed a $4.6-\mathrm{cm}$ solid mass in the pancreatic tail and a $4.2-\mathrm{cm}$ multilocular cystic mass in the pancreatic head with multiple liver and lymphatic metastasis. Notably, two solid masses were detected in the gastric wall of the upper body and the antrum; both were separated from the primary pancreatic cancer and seemed to be located in the submucosal layer. Esophagogastroduodenoscopy revealed a submucosal tumor with a normal mucosa in the posterior wall of the upper body of the stomach, suggesting the gastric hematogenous metastasis of pancreatic cancer. The suspected diagnosis was unresectable pancreatic cancer with multiple metastases that was concomitant with the intraductal papillary mucinous neoplasm of the pancreas.
\end{abstract}

(C) 2016 The Author(s)

Published by S. Karger AG, Basel

\section{Introduction}

Pancreatic cancer is the most lethal of the common human malignancies and the fourth and fifth leading cause of cancer-related death in the United States and Japan, respectively 
$[1,2]$. Since the majority of cases of are diagnosed at the advanced or metastatic stages of disease, less than $20 \%$ of patients are eligible for potentially curative resection. Even when curative resection is performed, most patients have disease recurrence [3]. Thus, pancreatic cancer is generally treated with chemotherapy rather than surgery. Gemcitabine, which was the first standard treatment for unresectable pancreatic cancer, has only a modest survival benefit. Although new chemotherapeutic regimens, such as FOLFIRINOX and albumin-bound paclitaxel plus gemcitabine have been developed, there have been no substantial improvements in the survival rate of pancreatic cancer patients over the past 25 years, while there have been notable improvements in the survival of patients with other forms of cancer [2]. Further efforts to develop therapies for pancreatic cancer might be needed.

This lethal cancer often develops both hematogenous and lymphatic metastases. The most frequent site of hematogenous metastasis in pancreatic cancer is the liver; liver metastases are observed in about $60-80 \%$ of autopsied cases of pancreatic cancer [4, 5]. Regional lymph node metastasis, peritoneal dissemination and lung metastasis are also frequently detected $[4,5]$. In addition, pancreatic cancer often directly infiltrates the peripheral organs. The gastric involvement of pancreatic cancer is occasionally observed in pancreatic cancer patients, which results not from metastasis but from direct invasion [4]. Actually, hematogenous gastric metastasis was very rare. We herein describe a rare case of hematogenous gastric metastasis from pancreatic cancer.

\section{Case Presentation}

A 72-year-old Japanese male with a previous history of cerebral infarction, arrhythmia, and cholecystectomy due to cholelithiasis presented with a 1-week history of general fatigue, pollakiuria, and thirst in December 2014. An intraductal papillary mucinous neoplasm in the pancreatic head was also found during the preoperative assessment for cholecystectomy. Because hyperglycemia $(565 \mathrm{mg} / \mathrm{dl})$ was detected, the patient was diagnosed with diabetes mellitus and insulin was immediately administered. As the patient's hyperglycemia improved, his general fatigue, pollakiuria, and thirst disappeared. At first, he did not complain of any abdominal symptoms, and a physical examination showed unremarkable results, but back pain thereafter slowly appeared. A urinalysis revealed glycosuria but not proteinuria. A blood test showed a marked elevation of the patient's hemoglobin A1c level (11.8\%, range 4.6-6.2\%) and slightly decreased levels of hemoglobin (12.6 g/dl, range 13.6$18.3 \mathrm{~g} / \mathrm{dl}$ ) and amylase (36 U/l, range 39-134 U/l), but normal liver and renal function. However, his cancer antigen $19-9(381.6 \mathrm{U} / \mathrm{ml}$, range $0.0-37.0 \mathrm{U} / \mathrm{ml})$ and carcinoembryonic antigen $(6.6 \mathrm{ng} / \mathrm{ml}$, range $0.0-5.0 \mathrm{ng} / \mathrm{ml})$ levels were elevated. Because pancreatic cancer was suspected based on the rapid progression of diabetes mellitus and high level of cancer antigen 19-9, a radiological examination was also performed. Computed tomography revealed a $4.6-\mathrm{cm}$ solid mass in the pancreatic tail with ring enhancement and a $4.2-\mathrm{cm}$ multilocular cystic mass in the pancreatic head (fig. 1). In addition, three liver metastatic masses and three abdominal lymph node metastases with ring enhancement, which were similar to the tumor in the pancreatic tail, were detected. Therefore, we suspected unresectable pancreatic cancer with multiple liver metastases that was concomitant with intraductal papillary mucinous neoplasm of the pancreas. To get a second opinion, the patient was discharged and visited another hospital. He eventually came back to our hospital 1 month after the first administration. The patient's back pain worsened and his cancer antigen 19-9 level increased $(507.8 \mathrm{U} / \mathrm{ml}$, range $0.0-37.0 \mathrm{U} / \mathrm{ml})$ in comparison to his first presentation. We re- 
evaluated his disease using computed tomography, which revealed progression of the primary pancreatic cancer and increased numbers of liver and abdominal lymph node metastases. Notably, two solid masses were detected in the gastric wall of the upper body and the antrum. Both of them were separated from the primary pancreatic cancer and seemed to be located in the submucosal layer (fig. 2). Esophagogastroduodenoscopy revealed a submucosal tumor with normal mucosa in the posterior wall of the upper body of the stomach, suggesting the gastric hematogenous metastasis of pancreatic cancer (fig. 3). Pathological examinations, such as endoscopic ultrasound-guided fine needle aspiration or percutaneous liver biopsy, had not been performed because the patient declined additional examinations and due to the seemingly rapid progression of the disease. The patient was diagnosed with pancreatic cancer metastasis to the liver, lymph node and stomach based on the elevated level of cancer antigen 19-9 and on the computed tomography image findings.

Chemotherapy using S-1 was immediately administered. After one course, we changed the $\mathrm{S}-1$ to gemcitabine after computed tomography revealed disease progression. Chemotherapy was terminated after two courses due to the deterioration of the patient's condition. The patient died 5 months after the first presentation.

\section{Discussion}

We herein report a rare case of hematogenous gastric metastasis from pancreatic cancer. Unfortunately, we could not obtain any pathological findings because the patient refused to undergo such examinations and due to the rapid progression of the disease. Although a low-density tumor with a high level of cancer antigen 19-9 was compatible with pancreatic ductal adenocarcinoma, ring enhancement could be considered to be atypical. Ring enhancement is usually observed in metastatic cancer; however, we could not detect a primary lesion in an organ other than the pancreas. A previous study showed that the ring enhancement pattern predicts adenosquamous carcinoma of the pancreas, which suggested that our case might be adenosquamous carcinoma [6]. In any case, the tumor in the pancreatic tail was considered to be the primary lesion of pancreatic cancer that metastasized to the liver, lymph nodes and stomach. Gastric involvement due to the direct invasion of pancreatic cancer has occasionally been observed in pancreatic cancer patients [4], but gastric metastasis from pancreatic cancer is quite a rare event. There are five pathways of secondary involvement of the stomach: (1) direct invasion; (2) intraoperative seeding; (3) hematogenous metastasis; (4) lymphatic metastasis, and (5) intraluminal or intramural dissemination [7]. In our case, two masses in the gastric wall were detected. Because these masses were located in the submucosa and separated from the primary tumor, we diagnosed them as hematogenous metastases to the stomach.

Previous studies of autopsy cases with pancreatic cancer described that the liver, regional lymph nodes, peritoneum, and lungs were common metastatic sites of pancreatic cancer $[4,5]$. Because distinguishing between metastasis and direct invasion is difficult in extremely progressed stages of carcinoma, autopsy studies often diagnosed metastasis when cancer cells were histologically detected in an organ [8]. Indeed, in the studies that distinguished metastasis from direct invasion, gastric metastasis of pancreatic cancer was described as being unusual $[4,5]$.

Metastasis to the stomach is also a rare condition. The incidences of gastric metastasis in autopsy cases with and without benign diseases were reported to range from 0.2 to $0.8 \%$ and from 1.7 to $5.4 \%$, respectively [9]. The major primary tumors were lung, breast, and 
esophageal cancers, as well as melanoma $[9,10]$. A previous large autopsy analysis of 347 gastric metastases reported two gastric metastases from pancreatic cancer [10], indicating that the pancreas is a rare primary site of gastric metastasis.

\section{Statement of Ethics}

The authors have no ethical conflicts to disclose. Informed consent was obtained from the kin after the patient died.

\section{Disclosure Statement}

The authors declare that there are no conflicts of interest in association with this report.

\section{References}

1 Matsuno S, Egawa S, Fukuyama S, Motoi F, Sunamura M, Isaji S, Imaizumi T, Okada S, Kato H, Suda K, Nakao A, Hiraoka T, Hosotani R, Takeda K: Pancreatic cancer registry in Japan: 20 years of experience. Pancreas 2004;28:219-230.

2 Jemal A, Siegel R, Ward E, Hao Y, Xu J, Murray T, Thun MJ: Cancer statistics, 2008. CA Cancer J Clin 2008;58:71-96.

-3 Paulson AS, Tran Cao HS, Tempero MA, Lowy AM: Therapeutic advances in pancreatic cancer. Gastroenterology 2013;144:1316-1326.

4 Cubilla A, Fitzgerald PJ: Pancreas cancer. I. Duct adenocarcinoma. A clinical-pathologic study of 380 patients. Pathol Annu 1978;13(Pt 1):241-289.

5 Blastik M, Plavecz E, Zalatnai A: Pancreatic carcinomas in a 60-year, institute-based autopsy material with special emphasis of metastatic pattern. Pancreas 2011;40:478-480.

6 Imaoka H, Shimizu Y, Mizuno N, Hara K, Hijioka S, Tajika M, Tanaka T, Ishihara M, Ogura T, Obayashi T, Shinagawa A, Sakaguchi M, Yamaura H, Kato M, Niwa Y, Yamao K: Ring-enhancement pattern on contrast-enhanced CT predicts adenosquamous carcinoma of the pancreas: a matched case-control study. Pancreatology 2014;14:221-226.

7 Feczko PJ, Collins DD, Mezwa DG: Metastatic disease involving the gastrointestinal tract. Radiol Clin North Am 1993;31:1359-1373.

8 Matsuda Y, Hagio M, Naito Z, Ishiwata T: Clinicopathological features of 30 autopsy cases of pancreatic carcinoma. J Nippon Med Sch 2012;79:459-467.

\9 Campoli PM, Ejima FH, Cardoso DM, Silva OQ, Santana Filho JB, Queiroz Barreto PA, Machado MM, Mota ED, Araujo Filho JA, Alencar Rde C, Mota OM: Metastatic cancer to the stomach. Gastric Cancer 2006;9:19-25.

10 Oda, Kondo H, Yamao T, Saito D, Ono H, Gotoda T, Yamaguchi H, Yoshida S, Shimoda T: Metastatic tumors to the stomach: analysis of 54 patients diagnosed at endoscopy and 347 autopsy cases. Endoscopy. 2001;33:507-510. 


\section{Case Reports in Gastroenterology

\begin{tabular}{l|l}
\hline Case Rep Gastroenterol 2016;10:75-80 \\
\hline $10.1159 / 000444249$ & $\begin{array}{l}\text { @ 2016 The Author(s). Published by S. Karger AG, Basel } \\
\text { www.karger.com/crg }\end{array}$ \\
\hline
\end{tabular} \\ Sasajima et al.: Hematogenous Gastric Metastasis of Pancreatic Cancer}
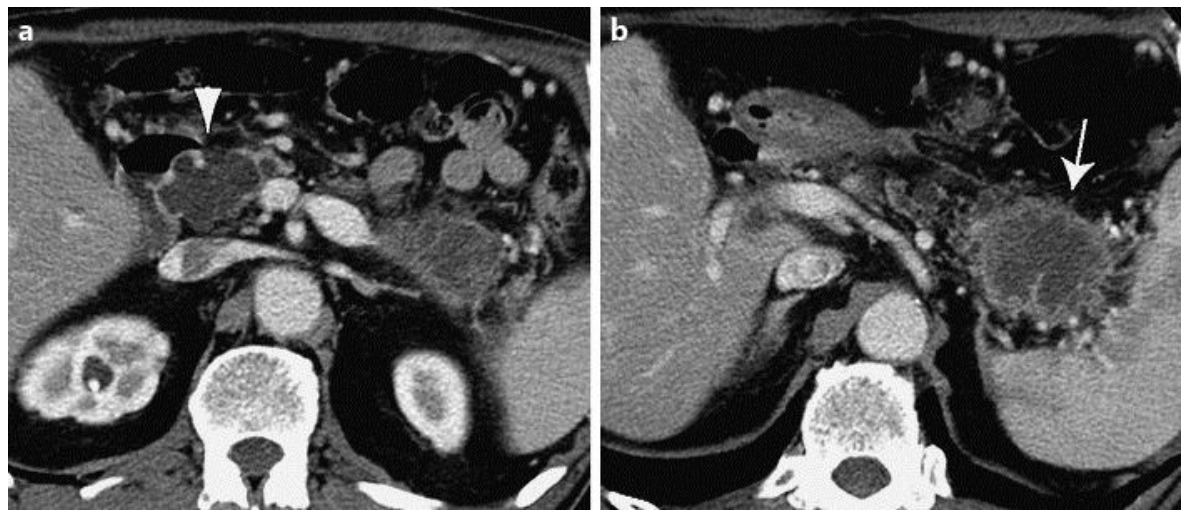

Fig. 1. Abdominal computed tomography at the first administration. a A $4.2-\mathrm{cm}$ multilocular cystic mass (arrowhead) was observed in the pancreatic head. b A 4.6-cm solid mass (arrow) was detected in the pancreatic tail.
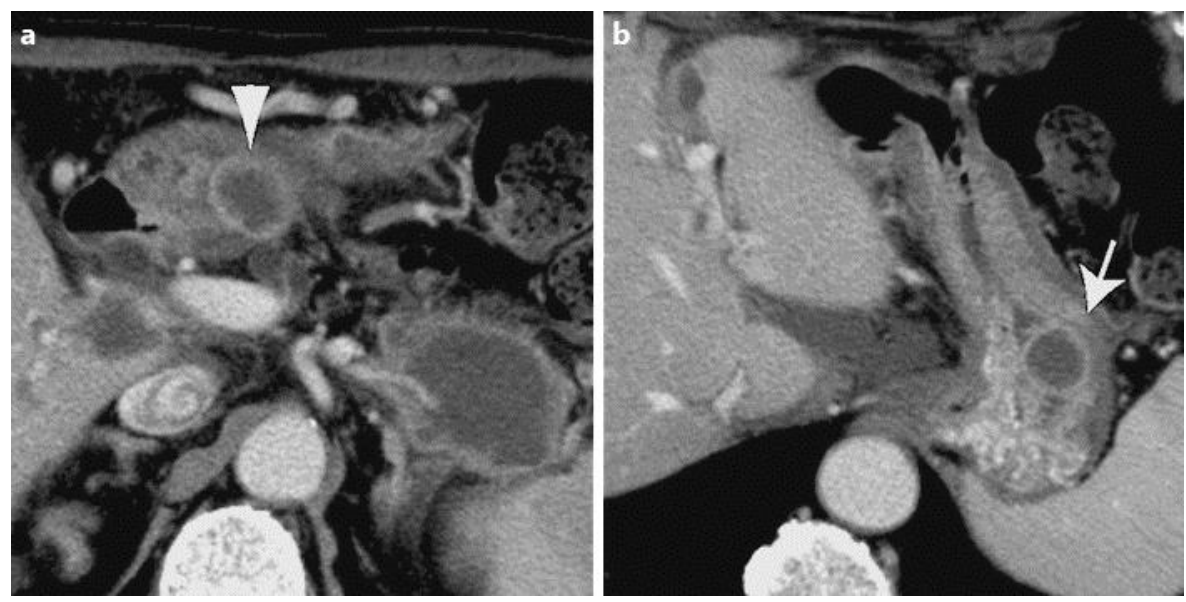

Fig. 2. Abdominal computed tomography 1 month after the first administration revealed two solid masses in the gastric wall of the antrum (a, arrowhead) and the upper body (b, arrow). 


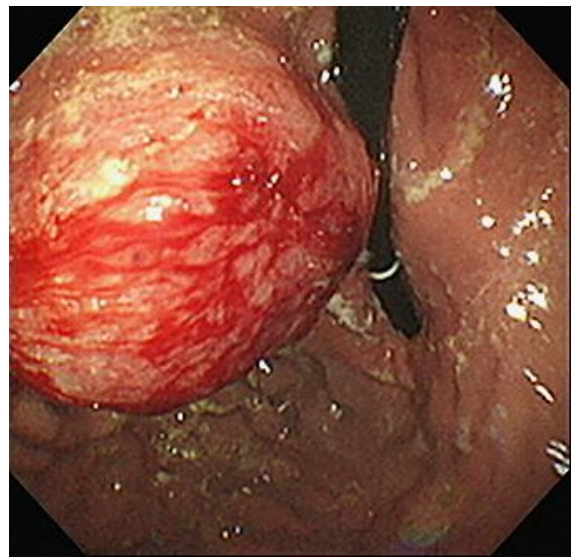

Fig. 3. Esophagogastroduodenoscopy revealed a submucosal tumor with normal mucosa in the posterior wall of the upper body of the stomach, suggesting the gastric hematogenous metastasis of pancreatic cancer. 